\title{
Existence of multidimensional phase transitions in a steady Van Der Waals flow
}

\author{
Shu-Yi Zhang \\ Communicated by Yuxi Zheng, received June 12, 2011.
}

\begin{abstract}
The purpose of this paper is to prove the existence of multidimensional subsonic phase transitions in a steady supersonic flow with the van der Waals type state function. The viscosity capillarity criterion [23] is applied to seek physical admissible planar waves in stead of the Lax entropy inequality [15], which is invalid under the subsonic condition. With the uniform stability result in $[\mathbf{2 6}]$, we shall proceed to establish the existence by performing the iteration scheme $[\mathbf{1 9}]$.
\end{abstract}

\section{Contents}

1. Introduction $\quad 79$

2. Admissible Criterion and the Main Problem 83

3. Linear Estimates 86

4. Compatibility conditions 88

5. Existence of the solution $\quad 91$

$\begin{array}{ll}\text { References } & 96\end{array}$

\section{Introduction}

In a fluid with non-monotonic state functions, say van der Waals fluid, nonlinear waves with different characteristic feature usually appear, such as shock waves, rarefaction waves, contact discontinuities and subsonic phase transitions, among which we shall be concerned with the subsonic phase transition in this paper. Roughly speaking, the subsonic phase transition is a piecewise smooth solution to the Euler

2000 Mathematics Subject Classification. 35L45, 35L50.

Key words and phrases. Supersonic flows, subsonic phase transitions, Euler equations.

Project 10901107 supported by National Natural Science Foundation of China. Project 11101076 supported by National Natural Science Foundation of China. Supported by Innovation Program of Shanghai Municipal Education Commission. 
equations with a single discontinuity, on both sides of which the sound speed is less than the fluid velocity in the normal direction to the discontinuity.

Due to the subsonic property, the well-known Lax entropy inequality for classical shock waves is violated. Hence, other admissible criterion is needed to assure the physical admissibility of subsonic phase transitions. There are several candidates available, among which the viscosity capillarity criterion is an important one. The viscosity capillarity criterion was first introduced by Slemrod [23] to study phase transitions in an unsteady van der Waals fluid. Ever since, the study of unsteady van der Waals fluid, especially on problems in one dimensional spaces have be carried out in many works. See $[\mathbf{1 0}],[\mathbf{1 6}],[\mathbf{2 2}],[\mathbf{2 3}]$ and references therein. There are also works concerning multidimensional problems in an unsteady van der Waals fluid. See [3], [4], [5], [25], [27] and references therein.

However, in contrast with the unsteady flow, the knowledge on steady van der Waals fluid is much less. In [26], the author proved the uniform stability of subsonic phase boundaries in multi-dimensional spaces by showing the validity of Lopatinski condition $[\mathbf{1 3}, \mathbf{1 8}]$. The purpose of this paper is to proceed to establish the existence of steady subsonic phase transitions. Since the stability result $[\mathbf{2 6}]$ indicates an $L^{2}$ energy estimate for the linearized problem, we can expect to establish the existence result by iteration as in $[\mathbf{1 9}]$. To complete the work, we will show the validity of compatibility conditions and fulfill the detail of the iteration.

The content of this paper is arranged as follows. The rest part of this section is a brief introduction to the concept of subsonic phase transition in a steady flow. In section 2, we will explain the viscosity-capillarity criterion and formulate the main problem for multi-dimensional phase transitions. In section 3 , the result of linear estimates in [26] will be presented. Section 4 and 5 mainly deal with the existence problem and the calculations of related compatibility conditions.

Let us briefly recall the equation of a steady van der Waals fluid and the concept of subsonic phase transitions.

The motion of an isothermal (or isentropic) 3-dimensional steady flow is governed by the following well-known Euler equations

$$
\left\{\begin{aligned}
\partial_{x}(\rho u)+\partial_{y}(\rho v)+\partial_{y}(\rho w)= & 0 \\
\partial_{x}\left(\rho u^{2}+p(\rho)\right)+\partial_{y}(\rho u v)+\partial_{y}(\rho u w) & =0 \\
\partial_{x}(\rho u v)+\partial_{y}\left(\rho v^{2}+p(\rho)\right)+\partial_{y}(\rho v w) & =0 \\
\partial_{x}(\rho u w)+\partial_{y}(\rho v w)+\partial_{y}\left(\rho w^{2}+p(\rho)\right) & =0
\end{aligned}\right.
$$

where $\rho$ is the density of the flow, $(u, v, w)^{T}$ is the velocity of the flow and $p$ is the pressure which is a function of $\rho$. Denote $U=(\rho, u, v, w)^{T}$,

$$
F_{0}(U)=\left(\begin{array}{c}
\rho u \\
\rho u^{2}+p \\
\rho u v \\
\rho u w
\end{array}\right), \quad F_{1}(U)=\left(\begin{array}{c}
\rho v \\
\rho u v \\
\rho v^{2}+p \\
\rho v w
\end{array}\right), \quad F_{2}(U)=\left(\begin{array}{c}
\rho w \\
\rho u w \\
\rho v w \\
\rho w^{2}+p
\end{array}\right)
$$


and

$$
\begin{aligned}
A_{1}(U) & =\left(\nabla_{U} F_{0}(U)\right)^{-1} \nabla_{U} F_{1}(U) \\
& =\frac{1}{\rho u\left(u^{2}-c^{2}\right)}\left(\begin{array}{cccc}
\rho u^{2} v & -\rho^{2} u v & \rho^{2} u^{2} & 0 \\
-u v c^{2} & \rho u^{2} v & -\rho u c^{2} & 0 \\
\left(u^{2}-c^{2}\right) c^{2} & 0 & \rho v\left(u^{2}-c^{2}\right) & 0 \\
0 & 0 & 0 & \rho v\left(u^{2}-c^{2}\right)
\end{array}\right), \\
A_{2}(U) & =\frac{1}{\rho u\left(u_{U}^{2}-c_{0}^{2}\right)}\left(\begin{array}{cccc}
\rho u^{2} w & -\rho^{2} u w & 0 & \rho^{2} u^{2} \\
-u w c^{2} & \rho u^{2} w & 0 & -\rho u c^{2} \\
0 & 0 & \rho w\left(u^{2}-c^{2}\right) & 0 \\
\left(u^{2}-c^{2}\right) c^{2} & 0 & 0 & \rho w\left(u^{2}-c^{2}\right)
\end{array}\right),
\end{aligned}
$$

where $c^{2}=d_{\rho} p(\rho)$ is the sound speed and the Euler equations (1.1) can be rewritten as

$$
\partial_{x} F_{0}(U)+\partial_{y} F_{1}(U)+\partial_{z} F_{2}(U)=0
$$

or

$$
\partial_{x} U+A_{1}(U) \partial_{y} U++A_{2}(U) \partial_{z} U=0 .
$$

When the flow is supersonic, namely

$$
u^{2}+v^{2}+w^{2}>c^{2}
$$

the system (1.1) is a hyperbolic conservation law, which is the case we are concerned with in this paper. In such case, nonlinear waves such as shock waves, rarefaction waves and contact discontinuities usually appear in a $\gamma$-pressure law flow. Rich literatures have been devoted to such topics and there still remain interesting open problems. See $[\mathbf{6}],[\mathbf{7}],[\mathbf{1 7}],[\mathbf{2 1}],[\mathbf{2 8}]$ and references therein.

However, in a van der Waals type flow, the above nonlinear waves are not all the cases, subsonic phase transitions usually appear due to the non-monotonicity of the state equation, which reads

$$
p(\tau)=\frac{R \theta}{\tau-b}-\frac{a}{\tau^{2}}
$$

where $\tau \equiv \rho^{-1}$ is the specific volume of the fluid, $\theta$ is the temperature of the fluid which is assumed to be a constant in an isothermal fluid, $R$ is the perfect gas constant and $a, b$ are positive constants. For $\theta \in(a /(4 b R), 8 a /(27 b R))$, the state equation (1.5) is not monotonic with respect to $\tau$. Precisely speaking, we can find $\tau_{*}, \tau^{*} \in(b,+\infty)$ such that

$$
\begin{cases}d_{\tau} p(\tau)<0 & \tau \in\left(b, \tau_{*}\right) \cup\left(\tau^{*},+\infty\right) \\ d_{\tau} p(\tau)>0 & \tau \in\left(\tau_{*}, \tau^{*}\right) .\end{cases}
$$

From the physical point of view, the fluid is in liquid phase for $\tau \in\left(b, \tau_{*}\right)$, while it is in vapor phase for $\tau \in\left(\tau^{*},+\infty\right)$. The state in the region $\left(\tau_{*}, \tau^{*}\right)$ is highly unstable, which doesn't actually exist in the real world [10].

A subsonic phase transition is a discontinuous solution to the Euler equation (1.1) with a single discontinuity, which changes phases across the discontinuity and satisfies certain subsonic condition on both sides of the discontinuity. To explain 
the concept with more detail, let us consider the following planar subsonic phase transition

$$
U(x, y, z)=\left\{\begin{array}{ll}
U_{-}=\left(\rho_{-}, u_{-}, v_{-}, w_{0}\right) & y<\sigma x \\
U_{+}=\left(\rho_{+}, u_{+}, v_{+}, w_{0}\right) & y>\sigma x
\end{array},\right.
$$

where $\rho_{ \pm}, u_{ \pm}, v_{ \pm}, w_{0}$ are constant states of the flow, $\sigma$ is the constant speed of the discontinuity $\{y=\sigma x\}$ and $\rho_{ \pm}$belong to different phases. The solution (1.7) satisfies the Rankine-Hugoniot condition

$$
\sigma\left[F_{0}(U)\right]-\left[F_{1}(U)\right]=0
$$

and the subsonic condition

$$
M_{ \pm}=\left|\frac{\sigma u_{ \pm}-v_{ \pm}}{c_{ \pm} \sqrt{1+\sigma^{2}}}\right|<1
$$

where [.] denotes the difference of a function across the discontinuity $\{y=\sigma x\}, M_{ \pm}$ and $c_{ \pm}^{2}=d_{\rho} p\left(\rho_{ \pm}\right)$are the Mach numbers and the sound speeds on each side of the discontinuity $\{y=\sigma x\}$ respectively. Denote by $u_{n \pm}=\left(\sigma u_{ \pm}-v_{ \pm}\right) / \sqrt{1+\sigma^{2}}$ and $u_{\tau}=\left(u_{ \pm}+\sigma v_{ \pm}\right) / \sqrt{1+\sigma^{2}}$ the normal velocity and the tangential velocity on each side of the discontinuity $\{y=\sigma x\}$ respectively, $j=\rho_{ \pm} u_{n \pm}$ the mass transfer flux, and $\pi=p_{ \pm}+j^{2} \tau_{ \pm}$. Then, the Rankine-Hugoniot condition (1.8) and the subsonic condition (1.9) can be simplified as

$$
[j]=0, \quad[\pi]=0, \quad\left[u_{\tau}\right]=0,
$$

and

$$
\left|\frac{u_{n \pm}}{c_{ \pm}}\right|<1 \quad \text { or } \quad\left|\frac{j^{2}}{\left.d_{\tau} p_{(} \tau_{ \pm}\right)}\right|<1,
$$

respectively. Like subsonic phase transitions in an unsteady fluid, those in a steady flow do not satisfy the Lax entropy inequality either. Considering the planar wave (1.7), we assume that the following supersonic condition is always valid in the coming arguments

$$
u_{ \pm}^{2}-c_{ \pm}^{2}>0
$$

Denote by

$$
\begin{aligned}
\lambda_{1}^{ \pm} & =\frac{1}{u_{ \pm}^{2}-c_{ \pm}^{2}}\left(u_{ \pm} v_{ \pm}-c_{ \pm} \sqrt{\triangle_{ \pm}}\right), \\
\lambda_{2}^{ \pm} & =\frac{v_{ \pm}}{u_{ \pm}}, \\
\lambda_{3}^{ \pm} & =\frac{1}{u_{ \pm}^{2}-c_{ \pm}^{2}}\left(u_{ \pm} v_{ \pm}+c_{ \pm} \sqrt{\triangle_{ \pm}}\right),
\end{aligned}
$$

the eigenvalues of $A_{1}\left(U_{ \pm}\right)$respectively with $\triangle_{ \pm}=u_{ \pm}^{2}+v_{ \pm}^{2}-c_{ \pm}^{2}$, which satisfy

$$
\lambda_{1}^{ \pm}<\lambda_{2}^{ \pm}<\lambda_{3}^{ \pm} \text {. }
$$

Obviously, the subsonic condition (1.9) yields the inequality

$$
\lambda_{1}^{ \pm}<\sigma<\lambda_{3}^{ \pm},
$$

which violates the Lax inequality for 1 st-shocks (3rd-shocks),

$$
\lambda_{1}^{+}<\sigma<\lambda_{1}^{-} \quad\left(\lambda_{3}^{+}<\sigma<\lambda_{3}^{-}\right) .
$$




\section{Admissible Criterion and the Main Problem}

In order to seek physical admissible solution, Slemrod [23] proposed the viscosity capillarity criterion for one dimensional unsteady fluids under Lagrange coordinates. Motivated by the study of multidimensional problems, Benzoni-Gavage $[3,4]$ applied this criterion to unsteady fluids under Euclid coordinates. In this section, let's recall the viscosity capillarity criterion to seek physical admissible phase transitions in a steady flow.

2.1. Viscosity capillarity criterion. Analogue to the traveling wave method for viscous shocks, the viscosity capillarity criterion picks the planar wave (1.7) which admits the existence of the following traveling wave

$$
U(\xi)=U\left(\frac{y-\sigma x}{\epsilon}\right)
$$

satisfying $U( \pm \infty)=U_{ \pm}$and the Navier-Stokes equations

$$
\left\{\begin{aligned}
\partial_{x}(\rho u)+\partial_{y}(\rho v)+\partial_{z}(\rho w) & =0 \\
\partial_{x}\left(\rho u^{2}+p(\rho)\right)+\partial_{y}(\rho u v)+\partial_{y}(\rho u w) & =\epsilon \nu \triangle u-\epsilon^{2} \partial_{x} \triangle\left(\rho^{-1}\right) \\
\partial_{x}(\rho u v)+\partial_{y}\left(\rho v^{2}+p(\rho)\right)+\partial_{y}(\rho v w) & =\epsilon \nu \triangle v-\epsilon^{2} \partial_{y} \triangle\left(\rho^{-1}\right) \\
\partial_{x}(\rho u w)+\partial_{y}(\rho v w)+\partial_{z}\left(w^{2}+p(\rho)\right) & =\epsilon \nu \triangle w-\epsilon^{2} \partial_{z} \triangle\left(\rho^{-1}\right)
\end{aligned}\right.
$$

where $\triangle=\partial_{x}^{2}+\partial_{y}^{2}+\partial_{z}^{2}$ is the Laplace operator, $\epsilon \nu$ is the viscosity coefficient and $\epsilon^{2}$ is the capillarity coefficient with $\epsilon \geq 0, \nu>0$. Substituting (2.1) into (2.2) and employing the Rankine-Hugoniot condition (1.10), we get the following hecteroclinic problem for the unknown function $\tau(\xi) \equiv 1 / \rho(\xi)$

$$
\left\{\begin{array}{l}
\tau^{\prime \prime}=\nu j \tau^{\prime}+\pi-p(\tau)-j^{2} \tau \\
\tau( \pm \infty)=\tau_{ \pm}
\end{array}\right.
$$

where the prime ' denotes the derivative of a function with respect to $\xi$. As in [4], the admissibility of subsonic phase transitions can be defined by

DEFINITION 2.1. The planar subsonic phase transition (1.7) is admissible if and only if the problem (2.3) has a solution. The solution $\tau(\xi)$ is called the viscosity capillarity profile or $\nu$-profile for simplicity. The pair $\left(\tau_{-}, \tau_{+}\right)$is called $\nu$-admissible.

One can find that the hecteroclinic problem (2.3) is exactly the same one for unsteady fluids [4]. Thus, the advantage of the known results in [4] is for us to take and let us have a brief recall. Denote by $\left\{\tau_{m}, \tau_{M}\right\}$ the Maxwell equilibrium defined by the equal area rule

$$
\int_{\tau_{m}}^{\tau_{M}}\left(p\left(\tau_{m}\right)-p(\tau)\right) d \tau=0
$$

Then, there exists $\tau_{1} \in\left(\tau_{M},+\infty\right)$ such that the chord connecting $\left(\tau_{1}, p\left(\tau_{1}\right)\right)$ and $\left(\tau_{m}, p\left(\tau_{m}\right)\right)$ is tangent to the graph of $p=p(\tau)$ at $\left(\tau_{1}, p\left(\tau_{1}\right)\right)$. With $\tau_{1}$ and $\tau_{m}$, we define

When $\nu=0$, the 0 -profile satisfies

$$
j_{1}^{2}=\frac{p\left(\tau_{1}\right)-p\left(\tau_{m}\right)}{\tau_{m}-\tau_{1}} .
$$

$$
\left\{\begin{array}{l}
\tau^{\prime \prime}=\pi-p(\tau)-j^{2} \tau \\
\tau( \pm \infty)=\tau_{ \pm}
\end{array}\right.
$$


As in [4], a 0 -profile $\bar{\tau}(\xi ; j)$ satisfying the first equation of $(2.4)$ can be found by the generalized equal area rule, which means

$$
\int_{\tau_{-}}^{\tau_{+}}\left(\pi-p(\tau)-j^{2} \tau\right) d \tau=0
$$

Moreover, for every $\bar{j}\left(0<\bar{j}^{2} \leq j_{1}^{2}\right)$, a unique pair $\left(\bar{\tau}_{-}(\bar{j}), \bar{\tau}_{+}(\bar{j})\right)$ can be found such that $\bar{\tau}_{-}$and $\bar{\tau}_{+}$can be connected by the 0 -profile with the parameters $j$. With the above results, Benzoni-Gavage [4] proved the structural stability and the existence of traveling waves for small $\nu$ by the center manifold method.

TheOREM 2.1. For $0<\bar{j}^{2} \leq j_{1}^{2}$, there exist $\nu_{0}>0$ and neighborhoods $\mathscr{J}_{0}, \mathscr{V}_{0}$ of $\bar{j},\left(\bar{\tau}_{-}(\bar{j}), \bar{\tau}_{+}(\bar{j})\right)$ respectively, such that, for $(j, \nu) \in \mathscr{J}_{0} \times\left[0, \nu_{0}\right]$, there are unique pair $\left(\tau_{-}, \tau_{+}\right) \in \mathscr{V}_{0}$, for which $\tau_{-}$and $\tau_{+}$are $\nu$ admissible with the parameters $j$.

Moreover, an additional jump condition can be derived from the above result for the subsonic phase transition (1.7). As we can see from the subsonic condition (1.9), a subsonic phase transition has one more characteristic going out of the free boundary than a shock wave. Hence, the Rankine-Hugoniot condition is not sufficient to guarantee the well-posedness of the corresponding initial boundary value problem. Nevertheless, the viscosity capillarity criterion can provide the following additional jump condition. By multiplying the equation in (2.3) with $\tau^{\prime}(\xi)$ and integrating from $-\infty$ to $+\infty$ with respect to $\xi$, it follows

$$
\left[f+\pi \tau-\frac{j^{2}}{2} \tau^{2}\right]=-\nu a(j, \nu)
$$

where $f=-\frac{a}{\tau}-R \theta \ln (\tau-b)$ is the free energy of the fluid and

$$
a(j, \nu)=j \int_{-\infty}^{+\infty}\left(\tau^{\prime}(\xi ; j, \nu)\right)^{2} d \xi
$$

with $\tau(\xi ; j, \nu)$ being the $\nu$-profile. Noticing $a(j, \nu)$ being a nonlocal term, the following lemma in $[\mathbf{3}]$ will be needed for linear estimate.

LEMMA 2.2. For all $\nu \in\left[0, \nu_{0}\right]$, the functions $a(j, \nu)$ is continuously differentiable. Moreover, its derivatives are continuous with respect to $\nu$ at $\nu=0$ and are bounded depending on the bounds of $U_{ \pm}$given in (1.7). There exists $\alpha>0$ such that for all $j \in \mathscr{J}$

$$
\lim _{\nu \rightarrow 0} \frac{\partial}{\partial j} a(j, \nu) \geq \alpha>0 .
$$

2.2. Problems, assumptions and main results. Compared with the unsteady fluid, in a steady supersonic flow, the variable $x$ can be regarded as the time variable [7]. Thus, endow the Euler equations with the initial data

$$
U(0, y, z)=\left\{\begin{array}{ll}
U_{-}^{0}(y, z) & y<\varphi_{0}(z) \\
U_{+}^{0}(y, z) & y>\varphi_{0}(z)
\end{array},\right.
$$

which changes phases across the discontinuity $\left\{y=\varphi_{0}(z)\right\}$ and satisfies certain compatibility conditions. We expect to construct the multidimensional subsonic phase transition

$$
U(x, y, z)= \begin{cases}U_{-}(x, y, z) & y<\varphi(x, z) \\ U_{+}(x, y, z) & y>\varphi(x, z)\end{cases}
$$


which satisfies the following nonlinear initial boundary value problem

$$
\left\{\begin{array}{lr}
\partial_{x} U_{ \pm}+A_{1}\left(U_{ \pm}\right) \partial_{y} U_{ \pm}+A_{2}\left(U_{ \pm}\right) \partial_{z} U_{ \pm}=0, & x>0, \pm(y-\varphi(x, z))>0 \\
\varphi_{x}\left[F_{0}(U)\right]-\left[F_{1}(U)\right]+\varphi_{z}\left[F_{2}(U)\right]=0, & y=\varphi(x, z) \\
{\left[I(\rho)+\frac{\left(\varphi_{x} u-v+\varphi_{z} w\right)^{2}}{2\left(1+\varphi_{x}^{2}+\varphi_{z}^{2}\right)}\right]=-\nu a(j, \nu),} & y=\varphi(x, z) \\
U_{ \pm}(0, y, z)=U_{ \pm}^{0}(y, z), \quad \varphi(0, z)=\varphi_{0}(z), &
\end{array}\right.
$$

where the second equation is the Rankine-Hugoniot condition, the third equation is a reformulation of the jump condition (2.5) with $I(\rho)=f+p \tau$ and $a(j, \nu)=$ $j \int_{-\infty}^{+\infty}\left(\tau^{\prime}(\xi ; j, \nu)\right)^{2} d \xi$ with $j=\rho_{ \pm}\left(\varphi_{x} u_{ \pm}-v_{ \pm}+\varphi_{z} w_{ \pm}\right) /\left.\sqrt{1+\varphi_{x}^{2}+\varphi_{z}^{2}}\right|_{y=\varphi(x, z)}$ and $\tau(\xi ; j, \nu)$ satisfying

$$
\left\{\begin{array}{l}
\tau^{\prime \prime}=\nu j \tau^{\prime}+\pi-p(\tau)-j^{2} \tau \\
\tau( \pm \infty)=\left.\tau_{ \pm}\right|_{y=\varphi(x, z)}
\end{array} .\right.
$$

Following Majda's approach [18], the following transformation

$$
\left\{\begin{aligned}
\widetilde{x} & =x \\
\widetilde{y} & = \pm(y-\varphi(x, z)), \quad \pm(y-\varphi(x, z))>0 \\
\widetilde{z} & =z \\
\widetilde{U}(\widetilde{x}, \widetilde{y}, \widetilde{z}) & =U(x, y, z)
\end{aligned}\right.
$$

maps the free boundary $\{y=\varphi(x, z)\}$ to the fixed one $\{\widetilde{y}=0\}$. Then the problem (2.9) becomes

$$
\left\{\begin{array}{lr}
\partial_{x} U_{ \pm} \pm\left(A_{1}\left(U_{ \pm}\right)-\varphi_{x} I-\varphi_{z} A_{2}\left(U_{ \pm}\right)\right) \partial_{y} U_{ \pm}+A_{2}\left(U_{ \pm}\right) \partial_{z} U_{ \pm}=0, & x, y>0 \\
\varphi_{x}\left[F_{0}(U)\right]-\left[F_{1}(U)\right]+\varphi_{z}\left[F_{2}(U)\right]=0, & y=0 \\
{\left[I(\rho)+\frac{\left(\varphi_{x} u-v+\varphi_{z} w\right)^{2}}{2\left(1+\varphi_{x}^{2}+\varphi_{z}^{2}\right)}\right]=-\nu a(j, \nu),} & y=0 \\
U_{ \pm}(0, y, z)=U_{ \pm}^{0}(y, z), \quad \varphi(0, z)=\varphi_{0}(z), &
\end{array}\right.
$$

where the tildes has been dropped for simplicity. We shall mainly deal with the above problem in the coming arguments.

For convenience, let's introduce several notations. Denote by $\omega$ the part of a neighborhood of the origin in $\{x=0, y>0\}, I=\bar{\omega} \cap\{y=0\}, \Omega \subset\{x, y>0\}$ a determinacy domain of $\omega$ with respect to the problem (2.11) with $\Omega_{T}=\Omega \cap\{x<T\}$ $\omega_{\tau}=\Omega \cap\{x=\tau\}$ and $\partial \Omega_{T}=\bar{\Omega}_{T} \cap\{y=0\}$.

To solve the problem (2.11), we propose the following assumptions:

(A1) For any fixed $\left(y_{0}, z_{0}\right) \in \Sigma_{0}=\left\{y=\varphi_{0}(z)\right\}$, there exists $\sigma\left(z_{0}\right) \in \mathbb{R}$ such that the problem (2.11) with the initial data frozen at $\left(y_{0}, z_{0}\right)$, admits a planar subsonic phase transition:

$$
U(x, y, z)= \begin{cases}U_{+}^{0}\left(\varphi_{0}\left(z_{0}\right), z_{0}\right), & x>\varphi_{0}\left(z_{0}\right)+\sigma\left(z_{0}\right) x \\ U_{-}^{0}\left(\varphi_{0}\left(z_{0}\right), z_{0}\right), & x<\varphi_{0}\left(z_{0}\right)+\sigma\left(z_{0}\right) x\end{cases}
$$

satisfying the viscosity-capillarity criterion.

(A2) For any fixed $s \geq 9, U_{ \pm}^{0} \in H^{s+1}(\omega), \varphi_{0} \in H^{s+3 / 2}(I)$ satisfy the higher order compatibility condition, which will be given in section 4.1, for $0 \leq k \leq s-1$.

The main result of this paper is 
THEOREM 2.3. Suppose that the initial data $\left(U_{ \pm}^{0}, \varphi_{0}\right)$ satisfies the assumptions (A1) and (A2), the problem (2.11) has a solution locally in time.

\section{Linear Estimates}

In this section we briefly recall the linear stability result of subsonic phase transitions in [26] and establish the energy estimate for the linear problem. First, let us derive the linearized problem of (2.11) and introduce the weighted Sobolev space.

3.1. Linearized problem. Consider the perturbation, $\left(U_{+}^{\epsilon}, U_{-}^{\epsilon}, \varphi^{\epsilon}\right)$, of the planar phase transition (1.7), which satisfies the problem (2.11) and

$$
\left.\left(U_{+}^{\epsilon}, U_{-}^{\epsilon}, \varphi^{\epsilon}\right)\right|_{\epsilon=0}=\left(U_{+}, U_{-}, \sigma x\right) .
$$

Denote

$$
\left(V_{+}, V_{-}, \psi\right)=\left.\frac{d}{d \epsilon}\left(U_{+}^{\epsilon}, U_{-}^{\epsilon}, \varphi^{\epsilon}\right)\right|_{\epsilon=0} .
$$

Then, the following linearized problem for the unknowns $\left(V_{+}, V_{-}, \psi\right)$ can be derived from (2.11),

$$
\left\{\begin{array}{lr}
\partial_{x} V_{ \pm} \pm\left(A_{1}\left(U_{ \pm}\right)-\sigma I\right) \partial_{y} V_{ \pm}+A_{2}\left(U_{ \pm}\right) \partial_{z} V_{ \pm}=f_{ \pm}, & x, y>0 \\
b_{0} \psi_{x}+b_{1} \psi_{z}+\mathscr{M}_{+} V_{+}+\mathscr{M}_{-} V_{-}=g, & y=0 \\
\left.\left(V_{+}, V_{-}, \psi\right)\right|_{x<0} & \text { vanish }
\end{array}\right.
$$

where

$$
\begin{gathered}
b_{0}=\left(\begin{array}{c}
{\left[F_{0}(U)\right]} \\
\frac{u_{\tau}}{1+\sigma^{2}}\left(\left[u_{n}\right]+\widetilde{\nu} \rho_{+}\right)
\end{array}\right), b_{1}=\left(\begin{array}{c}
{\left[F_{2}(U)\right]} \\
\frac{w_{0}}{\sqrt{1+\sigma^{2}}}\left(\left[u_{n}\right]+\widetilde{\nu} \rho_{+}\right)
\end{array}\right), \\
\mathscr{M}_{+}=\left(\begin{array}{c}
\sigma F_{0}^{\prime}\left(U_{+}\right)-F_{1}^{\prime}\left(U_{+}\right) \\
l_{+}
\end{array}\right), \mathscr{M}_{-}=\left(\begin{array}{c}
-\sigma F_{0}^{\prime}\left(U_{-}\right)+F_{1}^{\prime}\left(U_{-}\right) \\
l_{-}
\end{array}\right),
\end{gathered}
$$

where

$$
\begin{aligned}
& l_{+}=\left(\frac{c_{+}^{2}+\widetilde{\nu} j}{\rho_{+}}, \frac{\sigma\left(u_{n+}+\widetilde{\nu} \rho_{+}\right)}{\sqrt{1+\sigma^{2}}},-\frac{u_{n+}+\widetilde{\nu} \rho_{+}}{\sqrt{1+\sigma^{2}}}, 0\right), \\
& l_{-}=\left(-\frac{c_{-}^{2}}{\rho_{-}},-\frac{\sigma u_{n-}}{\sqrt{1+\sigma^{2}}}, \frac{u_{n-}}{\sqrt{1+\sigma^{2}}}, 0\right) .
\end{aligned}
$$

with $u_{n \pm}=\left(\sigma u_{ \pm}-v_{ \pm}\right) / \sqrt{1+\sigma^{2}}, u_{\tau}=\left(u_{ \pm}+\sigma v_{ \pm}\right) / \sqrt{1+\sigma^{2}}, j=\rho_{ \pm} u_{n \pm}$ and $\widetilde{\nu}=\nu \partial_{j} a(j, \nu)$.

To establish the energy estimate for the problem (3.1), let us introduce the following weighted norms. For any $\lambda>0$ and integer $s \geq 0$, we denote

$$
\begin{aligned}
\langle g\rangle_{s, \lambda, T}^{2} & =\sum_{\alpha+\beta+\gamma=s} \int_{0}^{T} \int_{-\infty}^{+\infty} \lambda^{2 \alpha} e^{-2 \lambda x}\left|\partial_{z}^{\beta} \partial_{x}^{\gamma} g\right|^{2} d z d x \\
|f|_{s, \lambda, T}^{2} & =\sum_{k=0}^{s} \int_{0}^{\infty}\left\langle\partial_{y}^{k} f\right\rangle_{s-k, \lambda, T}^{2} d y
\end{aligned}
$$


and

$$
\begin{aligned}
& \left\|\left(V_{+}, V_{-}, \psi\right)\right\|_{s, \lambda, T}^{2}=\langle\psi\rangle_{s+1, \lambda, T}^{2}+\sum_{k=0}^{s}\left(\left\langle\frac{\partial^{j} V_{+}}{\partial x^{j}}\right\rangle_{s-j, \lambda, T}^{2}+\left\langle\frac{\partial^{j} V_{-}}{\partial x^{j}}\right\rangle_{s-j, \lambda, T}^{2}\right) \\
& +\lambda\left(\left|V_{+}\right|_{s, \lambda, T}^{2}+\left|V_{-}\right|_{s, \lambda, T}^{2}\right) .
\end{aligned}
$$

We will simply denote $\langle\cdot\rangle_{s, T},|\cdot|_{s, T},\|\cdot\|_{s, T}$ the cases when the above norms are independent of $\lambda$ and $\langle\cdot\rangle_{s},\left.|\cdot|\right|_{s},\|\cdot\|_{s}$ the case $T=+\infty$.

3.2. Linear estimates. Let us recall the first main result of $[\mathbf{2 6}]$. For the problem (3.1), we have

THEOREM 3.1. There exists $\nu_{1}>0$ depending on the bounds of $U_{ \pm}$and $\sigma$, such that for $0<\nu<\nu_{1}$, the subsonic phase transition (1.7) is stable with respect to perturbations in the $y$-direction, which means the problem (3.1) without the variable $z$ being well-posed.

Denote by $V=\left(V_{+}, V_{-}\right)^{T}$ and

$$
\widehat{V}(s, \omega, y)=\frac{1}{(2 \pi)^{2}} \int_{0}^{\infty} \int_{-\infty}^{\infty} e^{-(s x+i \omega z)} V(x, y, z) d z d x
$$

the Laplace-Fourier transform of $V$ in $(x, z)$ with Res $>0$. Then from (3.1), we know that $\widehat{V}$ satisfies

$$
\frac{\partial \widehat{V}}{\partial y}=B(s, \omega) \widehat{V}+\widehat{f}
$$

where

$$
\begin{array}{cc}
B(s, \omega)= \\
\left(\begin{array}{cc}
-\left(A_{1}\left(U_{+}\right)-\sigma I\right)^{-1}\left(s I+i \omega A_{2}\left(U_{+}\right)\right) & 0 \\
0 & \left(A_{1}\left(U_{-}\right)-\sigma I\right)^{-1}\left(s I+i \omega A_{2}\left(U_{-}\right)\right)
\end{array}\right)
\end{array}
$$

and $\widehat{f}=\left(\left(A_{1}\left(U_{+}\right)-\sigma I\right)^{-1} \widehat{f}_{+},-\left(A_{1}\left(U_{-}\right)-\sigma I\right)^{-1} \widehat{f}_{-}\right)^{T}$.

Denote by $\left\{\lambda_{j}\right\}_{j=1}^{l}$ all distinct eigenvalues of $B(s, \omega)$ with multiplicity being $m_{j}$. Obviously, we have

$$
\mathbb{C}^{8}=\bigoplus_{j=1}^{l} \operatorname{Ker}\left[\left(\lambda_{j} I-B(s, \omega)\right)^{m_{j}}\right] .
$$

Introduce

$$
E^{+}(s, \omega)=\left\{w_{j} \in \operatorname{Ker}\left[\left(\lambda_{j} I-B(s, \omega)\right)^{m_{j}}\right] \mid \operatorname{Re} \lambda_{j}<0,1 \leq j \leq l\right\}
$$

the space of boundary values of all bounded solutions of the special form

$$
\widehat{V}(s, \omega, y)=\sum_{\operatorname{Re} \lambda_{j}<0} e^{\lambda_{j} y} \sum_{p=0}^{m_{j}-1} \frac{y^{p}}{p !}\left(\lambda_{j} I-B(s, \omega)\right)^{p} w_{j}
$$

to $(3.2)$ with $\widehat{f} \equiv 0$. The second main result of $[\mathbf{2 6}]$ is 
THEOREM 3.2. There exists $\nu_{1}>0$ depending on the bounds of $U_{ \pm}$and $\sigma$ given in (1.7) such that for $0<\nu<\nu_{1}$, the $\nu$-admissible subsonic phase transition (1.7) is uniformly stable, i.e. there exists $\eta>0$ such that the estimate

$$
\inf _{\substack{\Re s \geq 0 \\|s|^{2}+\omega^{2}=1}}\left|\left(b_{0} s+i b_{1} \omega\right) \mu+\mathscr{M}_{+} V_{+}+\mathscr{M}_{-} V_{-}\right|^{2} \geq \eta^{2}\left(\left|V_{+}\right|^{2}+\left|V_{-}\right|^{2}+\mu^{2}\right)
$$

holds for all $V=\left(V_{+}, V_{-}\right) \in E^{+}(s, \omega)$ and $\mu \in \mathbb{R}$.

Therefore, for the linear problem with variable coefficients (3.1), we can prove the following result by the same way as in [18]. Under assumption (A1) given at section 2.2 on the initial data $\left(U_{+}^{0}(y, z), U_{-}^{0}(y, z), \varphi_{0}(z)\right)$, there is $\delta>0$ such that for any smooth function $\left(U_{+}(x, y, z), U_{-}(x, y, z), \varphi(t, y)\right)$ satisfying

$$
\sup _{\bar{\Omega}_{T_{0}}}\left(\left|U_{ \pm}-U_{ \pm}^{0}\right|+\left|\varphi-\varphi_{0}\right|+\left|\varphi_{x}-\sigma(z)\right|+\left|\partial_{z}\left(\varphi-\varphi_{0}\right)\right|\right)<\delta
$$

the problem $(3.1)$ is well-posed for $\left(V_{+}, V_{-}, \psi\right)$, which means

THEOREM 3.3. Suppose that the assumption (A1) is satisfied and (3.4) holds for $\left(U_{+}, U_{-}, \varphi\right)$. If we have

$$
\left\{\begin{array}{l}
\left(f_{+}, f_{-}, g\right) \text { vanishes for } x<0 \text { and } x>T_{0} \\
\left|f_{+}\right|_{0}^{2}+\left|f_{-}\right|_{0}^{2}+\langle g\rangle_{0}^{2} \text { is finite }
\end{array}\right.
$$

then there is a unique strong solution $\left(V_{+}, V_{-}, \psi\right)$ to (3.1), and the estimate (3.6)

$$
\left\|\left(V_{+}, V_{-}, \psi\right)\right\|_{0, \lambda, T}^{2} \leq C_{1}\left(\frac{1}{\lambda}\left(\left|f_{+}\right|_{0, \lambda, T}^{2}+\left|f_{-}\right|_{0, \lambda, T}^{2}\right)+\langle g\rangle_{0, \lambda, T}^{2}\right), \quad 0 \leq T \leq T_{0}
$$

holds for $\lambda \geq C_{2}$, where $C_{1}, C_{2}>0$ depend only upon the quantities

$$
\left(\delta,\left|U_{+}, U_{-}, \varphi\right|_{s, T_{0}}\right)
$$

for any fixed $s \geq 9$.

Additionally, if $\left|f_{+}\right|_{s}^{2}+\left|f_{-}\right|_{s}^{2}+\langle g\rangle_{s}^{2}$ is finite for $s \geq 9$, and

$$
\left.\partial_{x}^{j} f_{ \pm}\right|_{y=0}=\left.\partial_{x}^{j} g\right|_{x=0}=0
$$

for any $0 \leq j \leq s-1$, then the solution $\left(V_{+}, V_{-}, \psi\right)$ belongs to

$$
H^{s} \times H^{s} \times H^{s+1}
$$

and satisfies

$$
\left\|\left(V_{+}, V_{-}, \psi\right)\right\|_{s, \lambda, T}^{2} \leq C_{1}\left(\frac{1}{\lambda}\left(\left|f_{+}\right|_{s, \lambda, T}^{2}+\left|f_{-}\right|_{s, \lambda, T}^{2}\right)+\langle g\rangle_{s, \lambda, T}^{2}\right), \quad 0 \leq T \leq T_{0} .
$$

\section{Compatibility conditions}

Before proving the existence, we have to derive the compatibility conditions for the initial data with which an approximate solution to the problem (2.11) can be constructed. 
4.1. Compatibility conditions. In order to derive the compatibility condition up to order $s-1$ with $s$ being a given positive integer, let us perform the formal Cauchy-Kowaleski computations. Assume that there is a smooth solution $\left(U_{+}, U_{-}, \varphi\right)$ to $(2.11)$. Then, from (A1) and (2.9), the zero-th order compatibility condition is that at $x=0$, the initial data satisfy

$$
\left\{\begin{array}{l}
\sigma\left[F_{0}\left(U^{0}\right)\right]-\left[F_{1}\left(U^{0}\right)\right]+\varphi_{0}^{\prime}\left[F_{2}\left(U^{0}\right)\right]=0 \\
{\left[I\left(\rho^{0}\right)+\frac{\left(\sigma u_{0}-v_{0}+\varphi_{0}^{\prime} w_{0}\right)^{2}}{2\left(1+\sigma^{2}+\varphi_{0}^{\prime 2}\right)}\right]=-\nu a\left(j_{0}, \nu\right)}
\end{array}\right.
$$

where

$$
j=\rho_{ \pm}^{0}\left(\sigma(z) u_{ \pm}^{0}-v_{ \pm}^{0}+\varphi_{0}^{\prime} w_{ \pm}^{0}\right) /\left.\sqrt{1+\sigma(z)^{2}+\varphi_{0}^{\prime 2}}\right|_{y=0}
$$

and $a\left(j_{0}, \nu\right)$ is defined similarly as in (2.5) with the parameter $j_{0}$.

Next, let us derive relations among $\left.\partial_{x}^{k+1} \varphi\right|_{x=0}$ and $\left.\partial_{y}^{k} U_{ \pm}^{0}\right|_{y=0}$. Differentiating the second and third equations of (2.9) with respect to $x$ at $x=y=0$, it follows

$$
\begin{gathered}
{\left[F_{0}\left(U^{0}\right)\right] \partial_{x}^{k+1} \varphi+F_{0}^{\prime}\left(U_{+}^{0}\right) \mathscr{A}_{+} \partial_{x}^{k} U_{+}+F_{0}^{\prime}\left(U_{-}^{0}\right) \mathscr{A}_{-} \partial_{x}^{k} U_{-}=g_{1}^{k}} \\
a_{0} \partial_{x}^{k+1} \varphi+l_{+}^{0} \partial_{x}^{k} U_{+}+l_{-}^{0} \partial_{x}^{k} U_{-}=g_{2}^{k}
\end{gathered}
$$

where $\mathscr{A}_{ \pm}= \pm\left(\sigma I-A_{1}\left(U_{ \pm}^{0}\right)+\varphi_{0}^{\prime} A_{2}\left(U_{ \pm}^{0}\right)\right), g_{1}^{k}$ and $g_{2}^{k}$ smoothly depend on

$$
\left\{\left.\partial_{z}^{l} \partial_{x}^{j} \varphi\right|_{x=0}: 0 \leq j \leq k, l+j \leq k+1\right\}
$$

and

$$
\left\{\left.\partial_{z}^{l} \partial_{x}^{j} U_{ \pm}^{0}\right|_{y=0}: 0 \leq j \leq k-1, l+j \leq k\right\},
$$

and

$$
\begin{aligned}
& a_{0}=\frac{u_{\tau}}{1+\sigma^{2}+\varphi_{0}^{\prime 2}}\left(\left[u_{n}\right]+\widetilde{\nu} \rho_{+}\right), \\
& l_{+}^{0}=\left(\frac{c_{+}^{2}+\widetilde{\nu} j}{\rho_{+}}, \frac{\sigma\left(u_{n+}+\widetilde{\nu} \rho_{+}\right)}{\sqrt{1+\sigma^{2}+\varphi_{0}^{\prime 2}}},-\frac{u_{n+}+\widetilde{\nu} \rho_{+}}{\sqrt{1+\sigma^{2}+\varphi_{0}^{\prime 2}}}, 0\right), \\
& l_{-}^{0}=\left(-\frac{c_{-}^{2}}{\rho_{-}},-\frac{\sigma u_{n-}}{\sqrt{1+\sigma^{2}+\varphi_{0}^{\prime 2}}}, \frac{u_{n-}}{\sqrt{1+\sigma^{2}+\varphi_{0}^{\prime 2}}}, 0\right)
\end{aligned}
$$

with $u_{n \pm}=\left(\sigma u_{ \pm}-v_{ \pm}+\varphi_{0}^{\prime} w_{ \pm}\right) / \sqrt{1+\sigma^{2}+\varphi_{0}^{\prime 2}}, u_{\tau}=\left(u_{ \pm}+\sigma v_{ \pm}\right) / \sqrt{1+\sigma^{2}+\varphi_{0}^{\prime 2}}$, $j=\rho_{ \pm} u_{n \pm}$ and $\widetilde{\nu}=\nu \partial_{j} a(j, \nu)$. On the other hand, from the equations of $U_{ \pm}$in (2.9) it follows

$$
\left.\partial_{x}^{k} U_{ \pm}\right|_{x=0}=\mathscr{A}_{ \pm}^{k} \partial_{y} U_{ \pm}^{0}+h_{ \pm}^{k},
$$

where $h_{ \pm}^{k}$ smoothly depend on

$$
\left\{\left.\partial_{z}^{l} \partial_{x}^{j} \varphi\right|_{x=0}: 0 \leq j \leq k, l+j \leq k+1\right\}
$$

and

$$
\left\{\left.\partial_{z}^{l} \partial_{x}^{j} U_{ \pm}^{0}\right|_{y=0}: 0 \leq j \leq k-1, l+j \leq k\right\} .
$$

Substituting (4.4) into (4.2) and (4.3), we obtain the following $k$-th order compatibility conditions for the problem (2.9) at $x=y=0$

$$
\left\{\begin{aligned}
{\left[F_{0}\left(U^{0}\right)\right] \partial_{x}^{k+1} \varphi+F_{0}^{\prime}\left(U_{+}^{0}\right) \mathscr{A}_{+}{ }^{k+1} \partial_{y}^{k} U_{+}^{0}+F_{0}^{\prime}\left(U_{-}^{0}\right) \mathscr{A}_{-}{ }^{k+1} \partial_{y}^{k} U_{-}^{0} } & =f_{1}^{k} \\
a_{0} \partial_{x}^{k+1} \varphi+l_{+}^{0} \mathscr{A}_{+}{ }^{k} \partial_{y}^{k} U_{+}^{0}+l_{-}^{0} \mathscr{A}_{-}{ }^{k} \partial_{y}^{k} U_{-}^{0} & =f_{2}^{k},
\end{aligned}\right.
$$


where $f_{1}^{k}$ and $f_{2}^{k}$ smoothly depend on $\left\{\left.\partial_{z}^{l} \partial_{x}^{j} \varphi\right|_{x=0}: 0 \leq j \leq k, l+j \leq k+1\right\}$ and $\left\{\left.\partial_{z}^{l} \partial_{x}^{j} U_{ \pm}^{0}\right|_{y=0}: 0 \leq j \leq k-1, l+j \leq k\right\}$.

4.2. Initial data satisfying the compatibility conditions. As in [19], here we show that there exist large classes of initial data satisfying the compatibility conditions. By simple calculation, we get the eigenvalues of $\left(A_{1}\left(U_{ \pm}^{0}\right)-\right.$ $\left.\varphi_{0}^{\prime}(z) A_{2}\left(U_{ \pm}^{0}\right)-\sigma(z) I\right)\left.\right|_{x=0}$ as the following

$$
\left\{\begin{array}{l}
\lambda_{1}^{ \pm}(z)=\frac{u_{ \pm}^{0} \tilde{v}_{ \pm}^{0}-c_{ \pm}^{0} \sqrt{\triangle_{ \pm}}}{u_{ \pm}^{2}-c_{ \pm}^{0}}-\sigma(z) \\
\lambda_{2}^{ \pm}(z)=\frac{\tilde{v}_{ \pm}^{0}}{u_{ \pm}^{0}-\sigma(z)} \\
\lambda_{3}^{ \pm}(z)=\frac{u_{ \pm}^{0} \tilde{v}_{ \pm}^{0}+c_{ \pm}^{0} \sqrt{\triangle_{ \pm}}}{u_{ \pm}^{2}-c_{ \pm}^{0}}-\sigma(z)
\end{array}\right.
$$

where $c_{ \pm}^{0}=\left(d_{\rho} p\left(\rho_{ \pm}^{0}\right)\right)^{\frac{1}{2}}, \tilde{v}_{ \pm}^{0}=v_{ \pm}^{0}-\varphi_{0}^{\prime} w_{ \pm}^{0}, \triangle_{ \pm}=\left(\tilde{v}_{ \pm}^{0}\right)^{2}+\left(\left(u_{ \pm}^{0}\right)^{2}-\left(c_{ \pm}^{0}\right)^{2}\right)\left(1+\left(\varphi_{0}^{\prime}\right)^{2}\right)$. Noting that $\lambda_{1}^{ \pm}$and $\lambda_{3}^{ \pm}$are of multiplicity $1, \lambda_{2}^{ \pm}$is of multiplicity 2 , we denote by $r_{1}^{ \pm}, r_{3}^{ \pm}$and $r_{2}^{ \pm}, \bar{r}_{2}^{ \pm}$the eigenvectors correspondingly.

Without loss of generality, we assume that the initial mass transfer flux

$$
j_{0}=\rho_{ \pm}^{0}\left(\sigma(z) u_{ \pm}^{0}-v_{ \pm}^{0}+\varphi_{0}^{\prime} w_{ \pm}^{0}\right) /\left.\sqrt{1+\sigma(z)^{2}+\varphi_{0}^{\prime 2}}\right|_{y=0}
$$

is positive, then from the subsonic condition (1.9) we have

$$
\lambda_{1}^{ \pm}(z)<0<\lambda_{2}^{ \pm}(z)<\lambda_{3}^{ \pm}(z) .
$$

Denote by $P^{+}(z)$ and $P^{-}(z)$ the smoothly varying projections onto the subspaces spanned by the eigenvectors associated with eigenvalues $\lambda_{2}^{+}(z), \lambda_{3}^{+}(z)$ and $\lambda_{1}^{-}(z)$ respectively. Similar to the Lemma 2.1 of Majda in [19], we have the following result:

Lemma 4.1. There exists $\nu_{0}$ depending on the bounds of $U_{ \pm}^{0}$ and $M$, such that for $0<\nu \leq \nu_{0}$, if $\left(v^{+}, v^{-}\right) \in \mathbb{R}^{4} \times \mathbb{R}^{4}$ satisfies

$$
P^{+} v^{+}=v^{+}, \quad P^{-} v^{-}=v^{-}
$$

and $\beta$ is constant, then from the identity

$$
G\left(\beta, v^{+}, v^{-}\right)=\beta\left(\begin{array}{c}
{\left[F_{0}\left(U^{0}\right]\right.} \\
a_{0}(y)
\end{array}\right)+\left(\begin{array}{c}
F_{0}^{\prime}\left(U_{+}^{0}\right) \mathscr{A}_{+}^{k+1} \\
l_{0}^{+}(y) \mathscr{A}_{+}^{k}
\end{array}\right) v^{+}+\left(\begin{array}{c}
F_{0}^{\prime}\left(U_{-}^{0}\right) \mathscr{A}_{-}^{k+1} \\
l_{0}^{-}(y) \mathscr{A}_{-}^{k-}
\end{array}\right) v^{-}=0
$$

we should have $\left(\beta, v^{+}, v^{-}\right)=0$.

Proof. The basis of the set

$$
\left\{\left(\beta, v^{+}, v^{-}\right) \mid P^{+} v^{+}=v^{+}, P^{-} v^{-}=v^{-}\right\}
$$

is given by

$$
(1,0,0) \cup\left(0, r_{2}^{+}, 0\right) \cup\left(0, \bar{r}_{2}^{+}, 0\right) \cup\left(0, r_{3}^{+}, 0\right) \cup\left(0, r_{1}^{-}, 0\right) .
$$


Obviously, we have

$$
\begin{aligned}
G(1,0,0) & =\left(\begin{array}{c}
{\left[F_{0}\left(U^{0}\right)\right]} \\
a_{0}
\end{array}\right), \\
G\left(0, r_{2}^{+}, 0\right) & =\left(\begin{array}{c}
\left(-\lambda_{2}^{+}\right)^{k+1} F_{0}^{\prime}\left(U_{+}^{0}\right) r_{2}^{+} \\
\left(-\lambda_{2}^{+}\right)^{k} l_{+}^{0} r_{2}^{+}
\end{array}\right), \\
G\left(0, \bar{r}_{2}^{+}, 0\right) & =\left(\begin{array}{c}
\left(-\lambda_{2}^{+}\right)^{k+1} F_{0}^{\prime}\left(U_{+}^{0}\right) \bar{r}_{2}^{+} \\
\left(-\lambda_{2}^{+}\right)^{k} l_{+}^{0} \bar{r}_{2}^{+}
\end{array}\right), \\
G\left(0, r_{3}^{+}, 0\right) & =\left(\begin{array}{c}
\left(-\lambda_{3}^{+}\right)^{k+1} F_{0}^{\prime}\left(U_{+}^{0}\right) r_{3}^{+} \\
\left(-\lambda_{3}^{+}\right)^{k} l_{+}^{0} r_{3}^{+}
\end{array}\right), \\
G\left(0,0, r_{1}^{-}\right) & =\left(\begin{array}{c}
\left(\lambda_{1}^{-}\right)^{k+1} F_{0}^{\prime}\left(U_{-}^{0}\right) r_{1}^{-} \\
\left(\lambda_{1}^{-}\right)^{k} l_{+}^{0} r_{1}^{-}
\end{array}\right) .
\end{aligned}
$$

Therefore, we get that the following determinant

$$
\begin{gathered}
\operatorname{det}\left(G(1,0,0), G\left(0, r_{2}^{+}, 0\right), G\left(0, \bar{r}_{2}^{+}, 0\right), G\left(0, r_{3}^{+}, 0\right), G\left(0,0, r_{1}^{-}\right)\right) \\
=\left(\left(\lambda_{2}^{+}\right)^{2} \lambda_{3}^{+} \lambda_{1}^{-}\right)^{k+1}\left|\begin{array}{ccccc}
{\left[F_{0}\left(U^{0}\right)\right]} & F_{0}^{\prime}\left(U_{+}^{0}\right) r_{2}^{+} & F_{0}^{\prime}\left(U_{+}^{0}\right) \bar{r}_{2}^{+} & F_{0}^{\prime}\left(U_{+}^{0}\right) r_{3}^{+} & F_{0}^{\prime}\left(U_{-}^{0}\right) r_{1}^{-} \\
a_{0} & -\frac{l_{+}^{0} r_{2}^{+}}{\lambda_{2}^{+}} & -\frac{l_{+}^{0} \bar{r}_{2}^{+}}{\lambda_{2}^{+}} & -\frac{l_{+}^{0} r_{3}^{+}}{\lambda_{3}^{+}} & \frac{l_{-}^{0} r_{1}^{-}}{\lambda_{1}^{-}}
\end{array}\right|
\end{gathered}
$$

does not vanish for sufficiently small $\nu$ and $K$ according to Theorem 3.1.

From the Lemma 4.1, now we can show that there exist large classes of initial data satisfying the compatibility conditions. As in [19], we have the following proposition

Proposition 4.2. Assume that $\left(V_{+}^{0}, V_{-}^{0}, \sigma\right) \in H^{s+\frac{1}{2}}(I)$ and $\varphi_{0} \in H^{2+\frac{3}{2}}(I)$ satisfy the zero-th order compatibility condition (4.1), and $g_{k}^{ \pm} \in H^{s+1-k}(I)(k \leq s-1)$ are arbitrary functions satisfying $P^{ \pm} g_{k}^{ \pm}=0$. Then there are $\left(U_{ \pm}^{0}(y, z), \varphi^{0}(x, z)\right) \in$ $H^{s+1}(\omega) \times H^{s+2}((-\infty, \infty) \times I)$ so that

(1)

$$
U_{ \pm}^{0}(0, z)=V_{ \pm}^{0}(z), \varphi^{0}(0, z)=\varphi_{0}(z), \partial_{x} \varphi^{0}(0, z)=\sigma(z)
$$

and

for $1 \leq k \leq s-1$;

$$
\left.\left(I-P^{ \pm}\right)\right)\left.\partial_{x}^{k} U_{ \pm}^{0}\right|_{x=0}=g_{k}^{ \pm}(z)
$$

(2) $\left(U_{ \pm}^{0}(y, z), \varphi^{0}(x, z)\right)$ satisfies the compatibility condition (4.5) for any $0 \leq$ $k \leq s-1$.

\section{Existence of the solution}

In this section, we prove the existence of the solution to the problem (2.11). For simplicity, let us denote the problem in the following abstract form

$$
\begin{cases}L^{ \pm}\left(U_{ \pm}, \varphi\right) U_{ \pm}=0, & x, y>0 \\ B\left(U_{+}, U_{-}, \varphi_{x}, \varphi_{z}\right)=0, & \text { on } y=0 \\ \left.U_{ \pm}\right|_{x=0}=U_{ \pm}^{0},\left.\quad \varphi\right|_{x=0}=\varphi_{0} & \end{cases}
$$

where

$$
L^{ \pm}\left(U_{ \pm}, \varphi\right)=\partial_{x} \pm\left(A_{1}\left(U_{ \pm}\right)-\varphi_{z} A_{2}\left(U_{ \pm}\right)-\varphi_{x} I\right) \partial_{y}+A_{2}\left(U_{ \pm}\right) \partial_{z}
$$

and $B(\cdot)=0$ represents the Rankine-Hugoniot condition and the viscosity-capillarity admissibility criterion given in (2.11). 
First let us construct a approximate solution to (5.1).

5.1. Approximate solutions. We are going to construct the following functions

$$
\tilde{U}_{ \pm}^{0} \in \bigcap_{j=0}^{s+1} C^{j}\left(\left[0, T_{0}\right], H^{s+1-j}\left(\omega_{t}\right)\right) \text { and } \varphi^{0} \in H^{s+2}\left(\Omega_{T_{0}}\right)
$$

such that

$$
\begin{cases}L^{ \pm}\left(\tilde{U}_{ \pm}^{0}, \varphi^{0}\right) \tilde{U}_{ \pm}^{0}=f_{ \pm}^{0}, & x, y>0 \\ B\left(\tilde{U}_{+}^{0}, \tilde{U}_{-}^{0}, \varphi_{x}^{0}, \varphi_{z}^{0}\right)=g_{1}^{0}, & \text { on } y=0 \\ \left.\tilde{U}_{ \pm}^{0}\right|_{x=0}=U_{ \pm}^{0},\left.\quad \varphi^{0}\right|_{x=0}=\varphi_{0} & \end{cases}
$$

and

$$
\left.\partial_{x}^{j} f_{ \pm}^{0}\right|_{x=0}=0,\left.\quad \partial_{x}^{j} g_{1}^{0}\right|_{x=0}=\left.\partial_{x}^{j} g_{2}^{0}\right|_{x=0}=0
$$

for $0 \leq j \leq s-1$.

Denoting

then from (5.3) and (5.4), we obtain

$$
m_{ \pm}^{j}=\left.\frac{\partial^{j} \tilde{U}_{ \pm}^{0}}{\partial x^{j}}\right|_{x=0}, \quad(0 \leq j \leq s)
$$

$$
m_{ \pm}^{j} \in H^{s+1-j} \quad(0 \leq j \leq s)
$$

Let $P\left(\partial_{t}, \partial_{x}, \partial_{y}\right)$ be a scalar linear hyperbolic operator of order $s+1, \tilde{m}_{ \pm}^{j} \in$ $H^{s+1-j}\left(\mathbb{R}^{2}\right)$ be an appropriate extension of $m_{ \pm}^{j}$ to $\{y<0\}$, and

$$
W_{ \pm}^{0} \in \bigcap_{j=1}^{s+1} C^{j}\left(\left[0, T_{0}\right], H^{s+1-j}\right)
$$

be the unique solution to the following Cauchy problem:

$$
\begin{cases}P W_{ \pm}^{0}=0, & x>0 \\ \left.\partial_{x}^{j} W_{ \pm}^{0}\right|_{x=0}=\tilde{m}_{ \pm}^{j}, & 1 \leq j \leq s .\end{cases}
$$

Then the restriction

$$
\tilde{U}_{ \pm}^{0}=\left.W_{ \pm}^{0}\right|_{x>0}
$$

together with $\varphi^{0} \in H^{s+2}\left(\Omega_{T_{0}}\right)$ given in Proposition 4.1 are the approximate solutions satisfying (5.3) (5.4). Indeed, since the initial data $\left(U_{ \pm}^{0}, \varphi_{0}\right)$ satisfy the compatibility conditions up to $s-1$, and from (4.5) we conclude (5.4).

5.2. The iteration scheme. Let us prove the existence of the solution to (5.1).

Denote by $E_{T}$ the extension operator given in the Lemma 3.1 of [19]. More precisely, for any fixed $0 \leq T \leq \frac{T_{0}}{2},\left(v_{+}, v_{-}, \varphi\right)$ satisfies $\left\|\left(v_{+}, v_{-}, \varphi\right)\right\|_{s, \lambda, T}<\infty$ and

$$
\begin{cases}\left.\partial_{x}^{j} v_{ \pm}\right|_{x=0}=0 & 0 \leq j \leq s-1 \\ \left.\partial_{x}^{j} \varphi\right|_{x=0}=0 & 0 \leq j \leq s\end{cases}
$$


the extended function $E_{T}\left(v_{+}, v_{-}, \varphi\right)$ satisfies

$$
\begin{cases}E_{T}\left(v_{+}, v_{-}, \varphi\right)=\left(v_{+}, v_{-}, \varphi\right) & \text { for } 0 \leq x \leq T \\ E_{T}\left(v_{+}, v_{-}, \varphi\right)=0 & \text { for } x<0 \text { and } x>T_{0} \\ \left\|E_{T}\left(v_{+}, v_{-}, \varphi\right)\right\|_{s_{1}, \lambda, T_{0}} \leq C_{s}\left\|\left(v_{+}, v_{-}, \varphi\right)\right\|_{s_{1}, \lambda, T} & \text { for any } 0 \leq s_{1} \leq s\end{cases}
$$

with a constant $C_{2}$ depending only on $s$.

As in [19], we introduce the iteration scheme for the problem (5.1). Let us define the functions inductively as the following:

$$
\left(\left(U_{+}^{n}, U_{-}^{n}, \varphi^{n}\right)=\left(\tilde{U}_{+}^{0}, \tilde{U}_{-}^{0}, \varphi^{0}\right)+E_{T_{n}}\left(W_{+}^{n}, W_{-}^{n}, \psi^{n}\right) \text { with }\left(W_{+}^{0}, W_{-}^{0}, \psi^{0}\right)=(0,0,0)\right.
$$

where $\left(\tilde{U}_{+}^{0}, \tilde{U}_{-}^{0}, \varphi^{0}\right)$ is the approximate solution constructed in section 5.1 and $\left(W_{+}^{n}, W_{-}^{n}, \psi^{n}\right)$ is the unique solution for $0 \leq t \leq T_{n}$ to the following problem provided $\left(W_{+}^{n-1}, W_{-}^{n-1}, \psi^{n-1}\right)$ being known already for $0 \leq x \leq T_{n-1}$ :

$$
\left\{\begin{array}{l}
L^{ \pm}\left(U_{ \pm}^{n-1}, \varphi^{n-1}\right) W_{ \pm}^{n}=f_{ \pm}^{n} \\
B_{\left(U_{+}^{n-1}, U_{-}^{n-1}, \varphi_{x}^{n-1}, \varphi_{z}^{n-1}\right)}^{\prime}\left(W_{+}^{n}, W_{-}^{n}, \psi_{x}^{n}, \psi_{z}^{n}\right)=g^{n} \\
\left(W_{+}^{n}, W_{-}^{n}, \psi^{n}\right) \text { vanish for } x<0
\end{array}\right.
$$

where $B_{\left(U_{+}, U_{-}, \varphi_{x}, \varphi_{z}\right)}^{\prime}\left(W_{+}, W_{-}, \psi_{x}, \psi_{z}\right)$ denotes the Fréchet derivatives of $B_{k}$ with respect to their arguments at $\left(U_{+}, U_{-}, \varphi_{x}, \varphi_{z}\right)$, i.e.

and

$$
\begin{aligned}
& B_{\left(U_{+}, U_{-}, \varphi_{x}, \varphi_{z}\right)}^{\prime}\left(W_{+}, W_{-}, \psi_{x}, \psi_{z}\right)= \\
& \left.\frac{d}{d \epsilon} B\left(U_{+}+\epsilon W_{+}, U_{-}+\epsilon W_{-}, \varphi_{x}+\epsilon \psi_{x}, \varphi_{z}+\epsilon \psi_{z}\right)\right|_{\epsilon=0},
\end{aligned}
$$

$$
\begin{aligned}
f_{ \pm}^{n}=\left\{\begin{array}{ll}
-L^{ \pm}\left(U_{ \pm}^{n-1}, \varphi^{n-1}\right) \tilde{U}_{ \pm}^{0}, & x>0 \\
0, & x<0
\end{array},\right. \\
g^{n}=G_{\left(U_{+}^{n-1}, U_{-}^{n-1}, \varphi_{x}^{n-1}, \varphi_{z}^{n-1}\right)} \\
\left(E_{T_{n-1}} W_{+}^{n+1}, E_{T_{n-1}} W_{-}^{n+1},\left(E_{T_{n-1}} \varphi^{n+1}\right)_{x},\left(E_{T_{n-1}} \varphi^{n+1}\right)_{z}\right) \\
-G\left(U_{+}^{n-1}, U_{-}^{n-1}, \varphi_{x}^{n-1}, \varphi_{z}^{n-1}\right) .
\end{aligned}
$$

As in $[\mathbf{1 9}]$, we expect to obtain the existence result by using the uniform stability result, namely Theorem 3.3 .

For any fixed $s \geq 9$, we denote by $C_{s}$ the Sobolev embedding constant satisfying

$$
\left\|v_{+}\right\|_{L^{\infty}\left(\Omega_{T}\right)}+\left\|v_{-}\right\|_{L^{\infty}\left(\Omega_{T}\right)}+\|\varphi\|_{W^{1, \infty}\left(\partial \Omega_{T}\right)} \leq C_{s}\left\|\left(v_{+}, v_{-}, \varphi\right)\right\|_{s, T}
$$

for any $\left(v_{+}, v_{-}, \varphi\right) \in H^{s} \times H^{s} \times H^{s+1}$, and $\epsilon_{0}>0$ a small quantity such that $\left(U_{+}, U_{-}, \varphi\right)$ satisfies

$$
\left\|\left(U_{+}-U_{+}^{0}, U_{-}-U_{-}^{0}, \varphi-\varphi_{0}^{\prime}(y)-x \sigma(y)\right)\right\|_{s, T_{0}}^{2}<\epsilon_{0}
$$

we have the estimate (3.4) valid.

For the iteration scheme (5.8), let us define

$$
T_{n}^{\prime}=\min \left\{T \mid\left\|\left(W_{+}^{n}, W_{-}^{n}, \psi^{n}\right)\right\|_{s, T}^{2} \geq \epsilon_{0}\right\}
$$

and

$$
T_{n}=\min \left(\frac{T_{0}}{2}, T_{n}^{\prime}\right)
$$


We have the following two propositions to prove Theorem 2.3:

Proposition 5.1. (Boundness) For any fixed $s \geq 9$, and $\epsilon_{0}>0$ being given in (5.10), there are $\beta \in(0,1)$ and $T_{*}>0$ such that the solution sequence $\left(V_{+}^{n}, W_{-}^{n}, \psi^{n}\right)$ defined by (5.8) satisfies

$$
\left\|\left(W_{+}^{n}, W_{-}^{n}, \psi^{n}\right)\right\|_{s, \lambda\left(T_{*}\right), T_{*}}<\epsilon_{0} \quad(\forall n \in \mathbb{N})
$$

where $\lambda(T)=C_{0} T^{-\beta}$.

Proof. Let briefly recall the proof of Proposition 4.1 in [19]. We shall prove the estimate (5.12) by induction on $n$. Obviously, it is true for $n=0$. Assuming that (5.12) holds for the case $n-1$, we study the problem (5.9).

Employing Theorem 3.3 for (5.9), it follows

$$
\left\|\left(W_{+}^{n}, W_{-}^{n}, \psi^{n}\right)\right\|_{s, \lambda(T), T}^{2} \leq C_{1}\left(\frac{1}{\lambda(T)}\left(\left|f_{+}^{n}\right|_{s, \lambda(T), T}^{2}+\left|f_{-}^{n}\right|_{s, \lambda(T), T}^{2}\right)+\left\langle g^{n}\right\rangle_{s, \lambda(T), T}^{2}\right) .
$$

Without loss of generality, we consider the case $0<T_{0} \leq 1$, which yields

$$
e^{-2 C_{0}} \leq e^{-2 C_{0} x} T^{-\beta} \leq 1
$$

for any $0 \leq \beta \leq 1$ and $0 \leq x \leq T \leq T_{0} \leq 1$.

From the definition, we can easily deduce

$$
\left\{\begin{array}{l}
\left|f_{ \pm}^{n}\right|_{s, \lambda(T), T}^{2} \leq C_{2} \sum_{k=0}^{s} T^{-2 \beta(s-k)}\left|f_{ \pm}^{n}\right|_{k, T}^{2} \\
\left\langle g^{n}\right\rangle_{s, \lambda(T), T}^{2} \leq C_{2} \sum_{k=0}^{s} T^{-2 \beta(s-k)}\left\langle g^{n}\right\rangle_{k, T}^{2} \\
\left\|\left(W_{+}^{n}, W_{-}^{n}, \psi^{n}\right)\right\|_{s, T}^{2} \leq C_{2}\left\|\left(W_{+}^{n}, W_{-}^{n}, \psi^{n}\right)\right\|_{s, \lambda(T), T}^{2}
\end{array}\right.
$$

with an absolute constant $C_{2}>0$ when $\lambda(T)=C_{0} T^{-\beta}$.

Therefore, from (5.13) we get

$$
\begin{aligned}
& \left\|\left(W_{+}^{n}, W_{-}^{n}, \psi^{n}\right)\right\|_{s, T}^{2} \leq \\
& C_{2}\left(T^{\beta} \sum_{k=0}^{s} T^{-2 \beta(s-k)}\left(\left|f_{+}^{n}\right|_{k, T}^{2}+\left|f_{-}^{n}\right|_{k, T}^{2}\right)+\sum_{k=0}^{s} T^{-2 \beta(s-k)}\left\langle g^{n}\right\rangle_{k, T}^{2}\right) .
\end{aligned}
$$

On the other hand, we have the following interpolation inequality:

$$
\left\{\begin{array}{l}
\left|f_{ \pm}^{n}\right|_{k, T}^{2} \leq \tilde{C}_{s}\left|f_{ \pm}^{n}\right|_{k, T}^{2 k / s}\left|f_{ \pm}^{n}\right|_{0, T}^{2-2 k / s} \\
\langle g\rangle_{k, T}^{2} \leq \tilde{C}_{s}\langle g\rangle_{s, T}^{2 k / s}\langle g\rangle_{0, T}^{2-2 k / s}
\end{array}\right.
$$

for any $0 \leq T \leq T_{0} / 2,0 \leq k \leq s$ with $\tilde{C}_{s}$ depending only upon s.

From the assumption (A2), and the induction assumption on $\left(W_{+}^{n}, W_{-}^{n}, \psi^{n}\right)$, we have a constant $C\left(\epsilon_{0}\right)$ depending only upon $\epsilon_{0}>0$ such that

$$
\left|f_{ \pm}^{n}\right|_{s, T_{0}} \leq C\left(\epsilon_{0}\right)
$$

for $f_{ \pm}^{n}$ given in $(5.9)$.

Furthermore, by using $\left.f_{ \pm}^{n}\right|_{x<0}=0$ and $\left\|f_{ \pm}^{n}\right\|_{C^{1}\left(\Omega_{T_{0}}\right)} \leq C$, we have

$$
\left|f_{ \pm}^{n}\right|_{0, T}^{2} \leq C T^{3}
$$

Substituting (5.16) and (5.17) into (5.15), it follows

$$
\left|f_{ \pm}^{n}\right|_{k, T}^{2} \leq C T^{3-3 k / s} \quad(0 \leq k \leq s)
$$


which implies

$$
\sum_{k=0}^{s} T^{-2 \beta(s-k)}\left(\left|f_{+}^{n}\right|_{k, T}^{2}+\left|f_{-}^{n}\right|_{k, T}^{2}\right) \leq C\left(1+T^{3 / s-2 \beta}\right)
$$

when $0 \leq \beta \leq \frac{3}{2(s+1)}$

From the property of Newton iteration scheme, we get

$$
g^{n}=-B\left(\tilde{U}_{+}^{0}, \tilde{U}_{-}^{0}, \partial_{x} \varphi^{0}, \partial_{y} \varphi^{0}\right)+O\left(\left|E_{T_{n-1}}\left(W_{+}^{n-1}, W_{-}^{n-1}, \partial_{x} \psi^{n-1}, \partial_{z} \psi^{n-1}\right)\right|^{2}\right)
$$

which implies

$$
\left\langle g^{n}\right\rangle_{s, T}^{2} \leq C\left(\left\langle B\left(\tilde{U}_{+}^{0}, \tilde{U}_{-}^{0}, \partial_{x} \varphi^{0}, \partial_{z} \varphi^{0}\right)\right\rangle_{s, T}^{2}+\left\|\left(W_{+}^{n-1}, W_{-}^{n-1}, \psi^{n-1}\right)\right\|_{s, T}^{4}\right) .
$$

Similar to (5.18), we obtain

$$
\left\langle g^{n}\right\rangle_{0, T}^{2} \leq C T^{3}
$$

by using $\left.g^{n}\right|_{x=0}=0$ and $\left\|g^{n}\right\|_{C^{1}\left(\partial \Omega_{T}\right)} \leq C$.

Substituting (5.20) and (5.21) into (5.15), it follows

$$
\langle g\rangle_{k, T}^{2} \leq C T^{3-3 k / s}\left(\left\langle B\left(\tilde{U}_{+}^{0}, \tilde{U}_{-}^{0}, \partial_{x} \varphi^{0}, \partial_{z} \varphi^{0}\right)\right\rangle_{s, T}^{2}+\left\|\left(W_{+}^{n-1}, W_{-}^{n-1}, \psi^{n-1}\right)\right\|_{s, T}^{4}\right)^{k / s}
$$

which implies

$$
\sum_{k=0}^{s} T^{-2 \beta(s-k)}\left\langle g^{n}\right\rangle_{k, T}^{2} \leq
$$

$$
C\left(T^{3-2 \beta s}+\left\langle B\left(\tilde{U}_{+}^{0}, \tilde{U}_{-}^{0}, \partial_{x} \varphi^{0}, \partial_{z} \varphi^{0}\right)\right\rangle_{s, T}^{2}+\left\|\left(W_{+}^{n-1}, W_{-}^{n-1}, \psi^{n-1}\right)\right\|_{s, T}^{4}\right) .
$$

Substituting (5.19) and (5.22) into (5.14), we conclude

$$
\begin{aligned}
& \left\|\left(W_{+}^{n}, W_{-}^{n}, \psi^{n}\right)\right\|_{s, T}^{2} \leq C \\
& \left(T^{3-2 \beta s}+T^{\beta}+T^{3 / s-\beta}+\left\langle B\left(\tilde{U}_{+}^{0}, \tilde{U}_{-}^{0}, \partial_{x} \varphi^{0}, \partial_{z} \varphi^{0}\right)\right\rangle_{s, T}^{2}\right. \\
& \left.+\left\|\left(W_{+}^{n-1}, W_{-}^{n-1}, \psi^{n-1}\right)\right\|_{s, T}^{4}\right) .
\end{aligned}
$$

which implies that there exist $T_{*}>0, \epsilon_{0}>0$ such that if $\|\left(W_{+}^{n-1}, W_{-}^{n-1}, \psi^{n-1} \|_{s, T_{*}}^{2}<\right.$ $\epsilon_{0}$, we have

$$
\|\left(W_{+}^{n}, W_{-}^{n}, \psi^{n} \|_{s, T_{*}}^{2}<\epsilon_{0}\right.
$$

Proposition 5.2. (Convergence) Under the same assumption as in Proposition 5.1, there are constants $C_{1}, C_{2}>0$ depending only on $\delta$, such that for any $\lambda>C_{2}$ and $T \leq T_{*}$, we have

$$
\begin{aligned}
& \left\|\left(W_{+}^{n+1}, W_{-}^{n+1}, \psi^{n+1}\right)-\left(W_{+}^{n}, W_{-}^{n}, \psi^{n}\right)\right\|_{0, \lambda, T}^{2} \leq \\
& C_{1}\left(\frac{1}{\lambda}+T^{2}\right)\left\|\left(W_{+}^{n}, W_{-}^{n}, \psi^{n}\right)-\left(W_{+}^{n-1}, W_{-}^{n-1}, \psi^{n-1}\right)\right\|_{0, \lambda, T}^{2} .
\end{aligned}
$$


Proof. When $T \leq T_{*}$, we can omit $E_{T_{n-1}}$ in the problem (5.9), from which we know that $\left(W_{+}^{n+1}-W_{+}^{n}, W_{-}^{n+1}-W_{-}^{n}, \psi^{n+1}-\psi^{n}\right)$ satisfies the following problem

$$
\left\{\begin{array}{l}
L^{ \pm}\left(U_{ \pm}^{n}, \varphi^{n}\right)\left(W_{ \pm}^{n+1}-W_{ \pm}^{n}\right)=\tilde{f}_{ \pm}^{n+1}, \quad t, x>0 \\
B_{\left(U_{+}^{n}, U_{-}^{n}, \varphi_{x}^{n}, \varphi_{z}^{n}\right)}^{\prime}\left(W_{+}^{n+1}-W_{+}^{n}, W_{-}^{n+1}-W_{-}^{n}, \psi_{x}^{n+1}-\psi_{x}^{n}, \psi_{z}^{n+1}-\psi_{z}^{n}\right)=\tilde{g}^{n+1} \\
\left(W_{+}^{n+1}-W_{+}^{n}, W_{-}^{n+1}-W_{-}^{n}, \psi^{n+1}-\psi^{n}\right) \text { vanishes for } x<0
\end{array}\right.
$$

where

$$
\left\{\begin{array}{l}
\tilde{f}_{ \pm}^{n+1}=-L^{ \pm}\left(U_{ \pm}^{n}, \varphi^{n}\right) \tilde{U}_{ \pm}^{0}+L^{ \pm}\left(U_{ \pm}^{n-1}, \varphi^{n-1}\right) \tilde{U}_{ \pm}^{0} \\
+\left(L^{ \pm}\left(U_{ \pm}^{n-1}, \varphi^{n-1}\right)-L^{ \pm}\left(U_{ \pm}^{n}, \varphi^{n}\right)\right) W_{ \pm}^{n} \\
\tilde{g}^{n+1}=g^{n+1}-g^{n}+\left(B_{\left(U_{+}^{n-1}, U_{-}^{n-1}, \varphi_{x}^{n-1}, \varphi_{z}^{n-1}\right)}^{\prime}\right. \\
\left.-B_{\left(U_{+}^{n}, U_{-}^{n}, \varphi_{x}^{n}, \varphi_{z}^{n}\right)}^{\prime}\right)\left(W_{+}^{n}, W_{-}^{n}, \psi_{x}^{n}, \psi_{z}^{n}\right)
\end{array} .\right.
$$

Employing Theorem 3.3 for the problem (5.25), it yields

$$
\begin{aligned}
& \left\|\left(W_{+}^{n+1}-W_{+}^{n}, W_{-}^{n+1}-W_{-}^{n}, \psi^{n+1}-\psi^{n}\right)\right\|_{0, \lambda, T}^{2} \\
& \leq C_{1}\left(\frac{1}{\lambda}\left(\left|\tilde{f}_{+}^{n+1}\right|_{0, \lambda, T}^{2}+\left|\tilde{f}_{-}^{n+1}\right|_{0, \lambda, T}^{2}\right)+\left\langle\tilde{g}^{n+1}\right\rangle_{0, \lambda, T}^{2}\right) .
\end{aligned}
$$

On the other hand, from (5.26) we easily deduce

$$
\left\{\begin{array}{l}
\left|\tilde{f}_{ \pm}^{n+1}\right|_{0, \lambda, T}^{2} \leq C_{2}\left\|\left(W_{+}^{n}-W_{+}^{n-1}, W_{-}^{n}-W_{-}^{n-1}, \psi^{n}-\psi^{n-1}\right)\right\|_{0, \lambda, T}^{2} \\
\left\langle\tilde{g}^{n+1}\right\rangle_{0, \lambda, T}^{2} \leq C_{2} T^{2}\left\langle\left(W_{+}^{n}-W_{+}^{n-1}, W_{-}^{n}-W_{-}^{n-1}, \psi^{n}-\psi^{n-1}\right)\right\rangle_{0, \lambda, T}^{2}
\end{array}\right.
$$

when $T \leq T_{*}$ be using Proposition 5.1. Thus, we immediately conclude (5.24) from (5.27) and (5.28).

With the above two propositions, we can prove Theorem 2.3.

The proof of Theorem 2.3: From Proposition 5.2 we know that there are $T_{* *} \in$ $\left(0, T_{*}\right], \alpha \in(0,1)$ and $\lambda_{0}>0$ such that

$$
\begin{aligned}
& \left\|\left(W_{+}^{n+1}-W_{+}^{n}, W_{-}^{n+1}-W_{-}^{n}, \psi^{n+1}-\psi^{n}\right)\right\|_{0, \lambda_{0}, T_{* *}}^{2} \\
& \leq \alpha\left\|\left(W_{+}^{n}-W_{+}^{n-1}, W_{-}^{n}-W_{-}^{n-1}, \psi^{n}-\psi^{n-1}\right)\right\|_{0, \lambda_{0}, T_{* *}}^{2}
\end{aligned}
$$

From Proposition 5.1 we know that $\left(W_{+}^{n}, W_{-}^{n}, \psi^{n}\right)$ is bounded in $H^{s} \times H^{s} \times$ $H^{s+1}$ for $0 \leq x \leq T_{*}$. Thus we obtain $\left(W_{+}, W_{-}, \psi\right) \in H^{s}\left(\Omega_{T_{* *}}\right) \times H^{s}\left(\Omega_{T_{* *}}\right) \times$ $H^{s+1}\left(\partial \Omega_{T_{* *}}\right)$ such that

$$
\left\{\begin{array}{ll}
W_{ \pm}^{n} \rightarrow W_{ \pm} & \text {in } H^{r}\left(\Omega_{T_{* *}}\right) \\
\psi^{n} \rightarrow \psi & \text { in } H^{r+1}\left(\partial \Omega_{T_{* *}}\right)
\end{array} \quad \text { as } n \rightarrow+\infty\right.
$$

for any $0 \leq r<s$, and

$$
U_{ \pm}=\tilde{U}_{ \pm}^{0}+W_{ \pm}, \quad \varphi=\varphi^{0}+\psi
$$

are the solutions to $(5.1)$.

\section{References}

[1] Alinhac, S., Existence d'ondes de raréfaction pour des systèmes quasi-linéaires hyperboliques multidimensionnels, Comm. Partial Differential Equaitons 14(2) (1989) 173-230.

[2] Benzoni-Gavage, S., Nonuniqueness of phase transitions near the Maxwell line, Proc. Amer. Math. Soc. 127 (1999) 1183-1190.

[3] Benzoni-Gavage, S., Stability of multi-dimensional phase transitions in a van der Waals fluid, Nonlinear Analysis, T.M.A. 31 (1998) 243-263. 
[4] Benzoni-Gavage, S.: Stability of subsonic planar phase boundaries in a van der Waals fluid, Arch. Rational Mech. Anal. 150 (1999) 23-55.

[5] Benzoni-Gavage, S.,Freistühler,H.: Effects of Surface Tension on the Stability of Dynamical Liquid-Vapor Interfaces,Arch. Rational Mech. Anal. 174 (2004) 111C150.

[6] Chen, S., Global existence of supersonic flow past a curved convex wedge, J. Partial Differential Equation 11 (1998), 43-62.

[7] Courant, R., Friedrichs, K.O., Supersonic flow and shock waves, Springer-Verlag: New York, 1977.

[8] Coulombel, J.F.; Secchi, P., The stability of compressible vortex sheets in two space dimensions, Indiana Univ. Math. J. 53(4) (2004) 941-1012.

[9] Coulombel, J.F.; Secchi, P., Nonlinear compressible vortex sheets in two space dimensions, preprint.

[10] Fan, H. and Slemrod, M., Dynamic flows with liquid/vapor phase transitions. In Handbook of mathematical fluid dynamics, pp. 373-420 Vol. I, North-Holland, Amsterdam, (2002).

[11] Grinfeld, M., Nonisothermal dynamic phase transitions, Quarterly Appl. Math. XLVII (1989) 71-84.

[12] Hattori, H., The Riemann problem for a van der Waals fluid with entropy rate admissibility criterion nonisothermal case, Journal Diff. Equa. 65 (1986) 158-174.

[13] Kreiss, H. O., Initial boundary value problems for hyperbolic systems, Comm. Pure Appl. Math. 23 (1970) 227-298.

[14] Korteweg, D.J., Sur la forme que prennent le équation des fluides si l'on tient compte des forces capilaires par des variantions densité, Arch. Néer. Sci. Exactes Sér. II 6 (1901) 1-24.

[15] Lax, P.D., Hyperbolic systems of conservation laws. II, Comm. Pure Appl. Math. 10 (1957) 537-467.

[16] LeFloch, Ph., Propagating phase boundaries: formulation of the problem and existence via the Glimm method, Arch. Rational Mech. Anal. 123 (1993) 153-197.

[17] Lien, W. and Liu, T. P., Nonlinear stability of a self-similar 3-dimensional gas flow, Commun. Math. Phys. 204 (1999), 525-549.

[18] Majda, A., The stability of multi-dimensional shock fronts, Mem. Amer. Math. Soc. 275 (1983) 1-95.

[19] Majda, A., The existence of multi-dimensional shock fronts, Mem. Amer. Math. Soc. 281 (1983) 1-93.

[20] Métivier, G. Stability of multimensional shock fronts. In Advances in The Theory of Shock Waves, Vol. 47, PnDEA; Birkhäuser, Boston, 2001, 25-103.

[21] Morawetz, C. S., On a weak solution for transonic flow problem, Comm. Pure Appl. Math. 38 (1985), 423-443.

[22] Shearer, M., Nonuniqueness of admissible solutions of Riemann initial value problem for a system of conservation laws of mixed type, Arch. Rational Mech. Anal. 93 (1986) 45-59.

[23] Slemrod, M., Admissibility criteria for propagating phase boundaries in a van der Waals fluid, Arch. Rational Mech. Anal. 81 (1983) 301-315.

[24] Slemrod, M., Dynamic Phase Transitions in a van der Waals Fluid, J. Diff. Equations 52 (1984) 1-23.

[25] Wang, Y.-G. and Xin, Z., Stability and existence of multidimensional subsonic phase transitions, Acta Math. Appl. Sinica 19 (2003) 529-558.

[26] Zhang, S.-Y., Stability of multidimensional phase transitions in a steady van der Waals flow, Chinese Annals Math.(Ser.B) 29 (2008), 223-238.

[27] Zhang, S.-Y., Discontinuous solutions to the Euler equations in a van der Waals fluid, Appl. Math. Letters, 20 (2007) 170-176.

[28] Zhang, Y., Global existence of steady supersonic potential flow past a curved wedge with a piecewise smooth boundary, SIAM J. Math. Anal. 31 (1999), 166-183.

School of Science, Shanghai Institute of Technology, P.O. Box 125, 1255 Fengpao

RD., Shanghai 201418, P.R.China

E-mail address: zh_shuyi@yahoo.com.cn 